\title{
A SURVEY ON STABILITY ANALYSIS OF DISCRETE-TIME PIECEWISE AFFINE SYSTEMS
}

\author{
Pratik Biswas *, Pascal Grieder ${ }^{* *}$, Johan Löfberg **, \\ Manfred Morari** \\ * Department of Electrical Engineering \\ Stanford University, California, USA \\ pbiswas@stanford.edu \\ ** Automatic Control Laboratory \\ Swiss Federal Institute of Technology \\ ETH Zentrum, ETLI 22, CH-8092, Zürich, Switzerland \\ Phone: ++4116326145 , fax: ++4116321211 \\ grieder / loefberg / morari @control.ee.ethz.ch
}

\begin{abstract}
Piecewise affine (PWA) systems are useful models for describing nonlinear and hybrid systems. They also result from LTI systems subject to constrained optimal control. Recently, stability analysis of PWA systems has received increased interest since it can help to obtain explicit feedback control laws of low complexity (Grieder et al., 2003b; Grieder and Morari, 2003; Grieder et al., 2004). A wide range of methods with varying degrees of conservativeness are available, for analyzing stability of PWA systems. This survey introduces the most promising existing methods and adapts them to discrete-time PWA systems. Specifically, we investigate the computation of common quadratic, common quartic, piecewise affine, piecewise quadratic and piecewise quartic Lyapunov functions using linear programming, semi-definite programming and sum-of-squares techniques. Subsequently, the different methods are compared regarding the likelihood of finding a certificate of stability and computation run time. The objective is to provide the reader with a practical 'recipe' for analyzing PWA systems. To this end, an extensive case study is performed.
\end{abstract}

Copyright (C)2005 IFAC

Keywords: Piecewise Affine Systems, Stability, Predictive Control, Sum-of-Squares

\section{INTRODUCTION}

Piecewise affine (PWA) systems have attracted much interest in the research community since they provide a useful modelling framework for a large class of hybrid systems. Discrete-time PWA systems are equivalent to interconnections of linear systems and finite automata (Sontag, 1996), to linear complementary systems (Heemels et al., 2001) and also hybrid systems in mixed logical dynamical (MLD) form (Bemporad and Morari, 1999). The MLD form encompasses a large class of hybrid systems including linear hybrid dynamical systems, hybrid automata and some classes of discrete event systems. Software for MLD modelling is available from (Torrisi and Bemporad, 2004) and an algorithm to transform an MLD system into a discrete-time PWA system is given in (Bemporad et al., 2000; Bemporad, 2004). In addition to hybrid systems, PWA 
systems are a powerful tool for approximating non-linear systems (Sontag, 1981). Furthermore, LTI systems subject to constraints and linear or quadratic optimal control are constrained autonomous PWA systems (Bemporad et al., 2002).

It was shown how to use semidefinite programming (SDP) to compute piecewise quadratic (PWQ) Lyapunov functions for continuous-time PWA systems in (Johansson and Rantzer, 1998) and for discrete-time PWA systems in (FerrariTrecate et al., 2002; Feng, 2002; Grieder et al., 2003a). A detailed description of PWA and PWQ stability analysis schemes was later provided in (Johansson, 2002). In (Prajna and Papachristodoulou, 2003), the authors show how to apply sum-of-squares methods (Parrilo, 2003) to compute higher order (piecewise) polynomial Lyapunov functions for continuous-time PWA systems. Recently, stability analysis of discrete-time PWA systems has received an increased interest since efficient stability analysis schemes can assist in the computation of feedback controllers of low complexity, for constrained LTI or PWA systems (Grieder et al., 2003b; Grieder and Morari, 2003; Grieder et al., 2004).

At this stage, there is a wide range of tools available to analyze PWA systems with no clear 'best' method. While simple Lyapunov functions (e.g. common quadratic) may not exist, higher order piecewise Lyapunov functions (e.g. piecewise quartic) may not be computable even if they exist, because of the conservative problem formulation which is needed to pose the respective SDP. In addition, the run-time of the respective analysis problems may be prohibitive. Finally, it is not clear if the proposed Lyapunov functions (i.e. PWQ etc.) are strong enough for practical problems.

The objective of this paper is to survey and evaluate various approaches to analyze discretetime PWA systems in practice. First, the basic computational building blocks (e.g. reachability analysis) and assumptions (e.g. set invariance) will be established. Second, we show how to compute PWA, PWQ and (Piecewise) Polynomial Lyapunov functions for discrete-time PWA systems. All of these analysis schemes have been presented previously for continuous-time PWA systems. However, the computation schemes for PWA and higher order piecewise polynomial Lyapunov functions were not previously published for discrete-time PWA systems. Finally, in a detailed case study the various analysis methods will be compared with respect to the likelihood of finding a Lyapunov function and the computation runtime.

\section{STABILITY OF DISCRETE-TIME PWA SYSTEMS}

\subsection{Problem Statement}

PWA systems are defined by a series of affine systems where each affine dynamic is defined over a polyhedral set in the state-input space.

Definition 1. (Polyhedron). A convex set $\mathcal{P} \subseteq \mathbb{R}^{n}$ given as an intersection of a finite number of $q$ closed half-spaces $\mathcal{P} \triangleq\left\{x \in \mathbb{R}^{n} \mid H x \leq K\right\}$, is called polyhedron. Here $H \in \mathbb{R}^{q \times n}, K \in \mathbb{R}^{q}$.

Specifically, an autonomous discrete-time PWA system is described by

$$
x(k+1)=A_{r} x(k)+g_{r}, \text { if } x(k) \in \mathcal{P}_{r}, r \in \mathcal{R},
$$

where the currently active dynamic $r$ is defined by the polyhedron

$$
\mathcal{P}_{r} \triangleq\left\{x \in \mathbb{R}^{n} \mid H_{r} x \leq K_{r}\right\}
$$

and the index set $\mathcal{R} \triangleq\{1,2, \ldots, R\}$, where $R$ denotes the number of different dynamics. We will denote the set of states over which the PWA system (1) is defined as $S_{\mathrm{PWA}}=\bigcup_{r \in \mathcal{R}} \mathcal{P}_{r}$.

The standing assumption throughout this paper is that the autonomous PWA system does not contain overlapping regions $\mathcal{P}_{i}$, i.e. $\mathcal{P}_{i} \cap \mathcal{P}_{j}=\emptyset$ for $i \neq j$. However, if the system dynamics are continuous, it is possible for two regions $\mathcal{P}_{i}$ and $\mathcal{P}_{j}$ to share a common facet. The 'no-overlaps' condition is needed to ensure that the stateupdate equation is uniquely defined for all states. Furthermore, we assume the PWA partition to be invariant $\left(x(0) \in S_{\mathrm{PWA}} \Rightarrow x(k) \in S_{\mathrm{PWA}}, \forall k \geq\right.$ $0)$, since the notion of stability has no practical relevance if the state trajectory exits the defined state space $S_{\mathrm{PWA}}$.

The following theorems are based on (Vidyasagar, 1993, p. 267) and have been adapted to deal with the special case of autonomous PWA systems of type (1) subject to constraints:

Theorem 1. (Asymptotic Stability). The origin $x=$ 0 is asymptotically stable for the autonomous PWA system if there exists a function $V(x)$ : $S_{\mathrm{PWA}} \rightarrow \mathbb{R}$ with $0 \in S_{\mathrm{PWA}}$ and scalar coefficients $\alpha>0, \beta>0, \rho>0$ such that $\beta\|x\| \geq V(x) \geq$ $\alpha\|x\|$ and $V\left(A_{r} x+g_{r}\right)-V(x) \leq-\rho\|x\|, \forall x \in \mathcal{P}_{r}$, $\forall r \in \mathcal{R}$. Here, $\|\cdot\|$ denotes a vector norm.

Theorem 2. (Exponential Stability). The origin $x=$ 0 is exponentially stable for the autonomous PWA system if there exists a function $V(x): S_{\mathrm{PWA}} \rightarrow \mathbb{R}$ with $0 \in S_{\mathrm{PWA}}$ and scalar coefficients $\alpha>0$, $\beta>0, \rho>0$ and $p>1$ such that $\beta\|x\|^{p} \geq$ $V(x) \geq \alpha\|x\|^{p}$ and $V\left(A_{r} x+g_{r}\right)-V(x) \leq-\rho\|x\|^{p}$, $\forall x \in \mathcal{P}_{r}, \forall r \in \mathcal{R}$. Here, $\|\cdot\|$ denotes a vector norm. 


\subsection{Reachability Analysis}

When searching for a piecewise Lyapunov function $V(x)$ (e.g. PWA or PWQ), the explicit representation of the decay rate $V(x(k+1))-V(x(k))$ depends on the regions $\mathcal{P}_{i}, \mathcal{P}_{j}$ which contain $x(k)$ and $x(k+1)$, respectively. Therefore, a region transition map needs to be created in order to formulate the search for such a Lyapunov function (Grieder et al., 2003a). For computational efficiency, this reachability computation is split into two parts: First, the feasible transitions from region $i$ to $j$ are identified and subsequently the set of states $\mathcal{P}_{i j}$ which actually execute such a transition are computed.

Specifically, a transition map $\mathcal{T}$ is first created $\forall i, j \in\{1, \ldots, R\}$ according to

$T(i, j)=\left\{\begin{array}{l}1, \text { if } \exists x \in \operatorname{int}\left(\mathcal{P}_{i}\right), \text { s.t. } A_{i} x+g_{i} \in \mathcal{P}_{j}, \\ 0, \text { otherwise, }\end{array}\right.$ where $\operatorname{int}(\cdot)$ denotes the strict interior of a set. The matrix $T$ is then used to construct the set $\mathcal{T} \triangleq\{i, j \in \mathcal{R} \mid T(i, j)=1\}$.

Remark 1. In principle, one LP needs to be solved for each element of the transition map $T$, i.e. a total of $R^{2}$ LPs, where $R$ denotes the total number of system dynamics. However, instead of solving LPs directly, it is advisable to first compute bounding boxes (hyper-rectangles) for each region $\mathcal{P}_{r}(r \in \mathcal{R})$. In addition, a bounding box of the affine map of the region $\mathcal{P}_{r}^{+}=\left\{A_{r} x+\right.$ $\left.g_{r} \in \mathbb{R}^{n} \mid x \in \mathcal{P}_{r}\right\}$ needs to be computed. The number of LPs which need to be solved in order to compute the bounding boxes is linear in the number of regions $R$ and state space dimension $n$. This computation is tractable even for very complex partitions. The bounding boxes can be efficiently checked for intersections, such that certain transitions $i \rightarrow j$ can be ruled out. In our experience, the bounding box implementation is the most effective way to compute $\mathcal{T}$ for complex region partitions.

In a second step, the transition sets $\mathcal{P}_{i j}$ for system (1) are explicitly computed for all $i, j \in \mathcal{T}$ :

$$
\begin{aligned}
\mathcal{P}_{i j} & =\left\{x \in \mathbb{R}^{n} \mid x \in \mathcal{P}_{i}, \quad A_{i} x+g_{i} \in \mathcal{P}_{j}\right\} \\
& =\left\{x \in \mathbb{R}^{n} \mid H_{i j} x \leq K_{i j}\right\} .
\end{aligned}
$$

If $T(i, j)=0$, then $\mathcal{P}_{i j}=\emptyset$. The transition set $\mathcal{T}$ will be used in the following sections to search for piecewise Lyapunov functions.

\section{PWA LYAPUNOV FUNCTIONS}

It will be shown in the following how to formulate the search for a PWA Lyapunov function guaranteeing asymptotic stability for autonomous PWA systems as a linear program (LP). The scheme presented in this section is based on results for continuous time systems which were published in (Johansson, 2002). The computation scheme for the PWA Lyapunov function is non-conservative (i.e. if a PWA Lyapunov function over the given partition exists, it will be found), thus it may succeed when no PWQ Lyapunov function can be found with the schemes in (Ferrari-Trecate et al., 2002; Feng, 2002; Grieder et al., 2003a).

In each polyhedral cell $\mathcal{P}_{r}$, the function $\operatorname{PWA}(x)$ will be defined by $\operatorname{PWA}_{r}(x)=x^{T} L_{r}+C_{r}$. It should be pointed out that the PWA Lyapunov function is allowed to be discontinuous and/or non-convex, since we are dealing with discretetime systems. Hence, we are looking for a function $\operatorname{PWA}(x)$ which satisfies the following constraints:

$$
\begin{gathered}
\beta\|x\|_{1} \geq \operatorname{PWA}_{r}(x) \geq \alpha\|x\|_{1}, \\
\alpha, \beta>0, \forall x \in \mathcal{P}_{r}, \forall r \in \mathcal{R}, \\
\operatorname{PWA}_{j}\left(A_{i} x+g_{i}\right)-\operatorname{PWA}_{i}(x) \leq-\rho\|x\|_{1}, \\
\rho>0, \forall x \in \mathcal{P}_{i j}, \forall i, j \in \mathcal{T} .
\end{gathered}
$$

Note that it is possible to replace the 1-norm in (4) with any other linear norm, e.g. $\infty$-norm.

In order pose the search for $\operatorname{PWA}(x)$ as an LP, it is necessary to first compute the vertices of the transition sets $\left(\operatorname{vert}\left(\mathcal{P}_{i j}\right)\right)$ and of the dynamic sets $\left(\operatorname{vert}\left(\mathcal{P}_{i}\right)\right)$. The problem of finding a PWA Lyapunov function, for the autonomous PWA system (1) such that the conditions in Theorem 1 are satisfied can now be stated as

$$
\begin{gathered}
\beta\|x\|_{1} \geq \operatorname{PWA}_{r}(x) \geq \alpha\|x\|_{1}, \\
\alpha, \beta>0, \forall x \in \operatorname{vert}\left(\mathcal{P}_{r}\right), \forall r \in \mathcal{R}, \quad(5 \mathrm{a}) \\
\operatorname{PWA}_{j}\left(\tilde{A}_{i} x+\tilde{g}_{i}\right)-\operatorname{PWA}_{i}(x) \leq-\rho\|x\|_{1}, \\
\rho>0, \forall x \in \operatorname{vert}\left(\mathcal{P}_{i j}\right), \forall i, j \in \mathcal{T} . \quad(5 \mathrm{~b})
\end{gathered}
$$

Since the vertices of all sets $\mathcal{P}_{i}$ and $\mathcal{P}_{i j}$ are known, the resulting problem is linear in $L_{r}, C_{r}$ and can therefore be solved as an LP.

Remark 2. It follows from the constraints (5a) (i.e. $V(0)=0$ ) that the PWA Lyapunov function will have no offset term for all regions containing the origin, i.e. $C_{i}=0, \forall i \in \mathcal{R}_{0}$. Since the Lyapunov function is PWA for the remainder of the state space, there will always exist a parameter $\beta$ bounding the Lyapunov function from above. Hence, the 'upper bound' constraint $\beta\|x\|_{1} \geq$ $P W A_{i}(x)$ does not need to be enforced when solving the LP (5).

Theorem 3. (Asymptotic Stability via LP). If the LP (5) associated to the autonomous PWA system 
(1) is feasible, then this system is asymptotically stable.

Proof First note that the linear norm $\|x\|_{1}$ is convex. Since the function $\operatorname{PWA}_{r}(x), r \in \mathcal{R}$ is piecewise affine, it follows that satisfaction of $(5 \mathrm{a})$ for all vertices of $\mathcal{P}_{r}$ implies that the inequalities in (5a) will also hold $\forall x \in \mathcal{P}_{r}$. Furthermore, if (5b) holds for all vertices of $\mathcal{P}_{i j}$, it follows from linearity of the system dynamics (1) that the inequality will hold for all states $x \in \mathcal{P}_{i j}$. Since the partition $S_{\mathrm{PWA}}$ is invariant, it follows that $S_{\mathrm{PWA}}=\bigcup_{r \in \mathcal{R}} \mathcal{P}_{r}=\bigcup_{i, j \in \mathcal{T}} \mathcal{P}_{i j}$. Therefore, the inequalities in (5a) and (5b) hold $\forall x \in S_{\mathrm{PWA}}$ such that the conditions in Theorem 1 are satisfied, i.e. feasibility of (5) implies asymptotic stability of the autonomous PWA system (1).

It should be noted that the required computation time may become large due to the extensive reachability analysis (Section 2.2), vertex enumeration and size of the final LP. Specifically, the LP (5) introduces one constraint for each vertex of each region $\mathcal{P}_{r}, \forall r \in \mathcal{R}$ (see (5a)) and one constraint for each vertex of each $\mathcal{P}_{i j}, \forall i, j \in \mathcal{T}$ (see (5b)). The number of variables is $(n+1) R$, where $R$ denotes the number of dynamics and $n$ the state space dimension.

\section{PWQ LYAPUNOV FUNCTIONS}

It will be shown in the following how to formulate the search for common quadratic and PWQ Lyapunov functions guaranteeing asymptotic stability for autonomous PWA systems as an SDP problem. The use of SDP based methods is illustrated for continuous-time systems in (Johansson and Rantzer, 1998; Johansson, 2002) and for discrete-time systems in (Ferrari-Trecate et al., 2002; Feng, 2002; Grieder et al., 2003a). In each polyhedral cell $\mathcal{P}_{r}$, the function $\operatorname{PWQ}(x)$ is defined by $\mathrm{PWQ}_{r}(x)=x^{T} Q_{r} x+x^{T} L_{r}+C_{r}$. Specifically, this function should satisfy

$$
\begin{gathered}
\beta x^{T} x \geq \mathrm{PWQ}_{r}(x) \geq \alpha x^{T} x, \\
\alpha, \beta>0, \forall x \in \mathcal{P}_{r}, \forall r \in \mathcal{R}, \\
\mathrm{PWQ}_{j}\left(A_{i} x+g_{i}\right)-\mathrm{PWQ}_{i}(x) \leq-\rho x^{T} x, \\
\rho>0, \forall x \in \mathcal{P}_{i j}, \forall i, j \in \mathcal{T} .
\end{gathered}
$$

Let $G_{i j}(x)=K_{i j}-H_{i j} x$ (recall the notation in (3)) and $\Delta V_{i j}(x)=\mathrm{PWQ}_{j}\left(A_{i} x+g_{i}\right)-\mathrm{PWQ}_{i}(x)$. By applying the $S$-procedure ${ }^{1}$ (Boyd et al., 1994), we can conservatively approximate (6b) with

$\exists N_{i j} \geq 0: \Delta V_{i j}(x) \leq-\rho x^{T} x-G_{i j}^{T}(x) N_{i j} G_{i j}(x)$,

$1 f(x) \geq 0 \forall x: g_{i}(x) \geq 0$ is conservatively replaced with the sufficient condition $\exists \lambda_{i} \geq 0: f(x) \geq \sum \lambda_{i} g_{i}(x)$ where $\rho>0$ and $N_{i j}$ is an arbitrary symmetric matrix consisting of non-negative elements only.

With $\bar{x}=\left[\begin{array}{ll}x & 1\end{array}\right]^{T}$ with $x \in \mathcal{P}_{i j}$, we arrive at the following inequality (Johansson and Rantzer, 1998; Ferrari-Trecate et al., 2002):

$$
\begin{aligned}
& \Delta V_{i j}(x)=\bar{x}^{T}\left[\begin{array}{cc}
\Delta Q_{i j} & \Delta L_{i j}, \\
\Delta L_{i j}^{T} & \Delta C_{i j}
\end{array}\right] \bar{x} \\
& \leq \bar{x}^{T}\left(-\left[\begin{array}{c}
-H_{i j}^{T} \\
K_{i j}^{T}
\end{array}\right] N_{i j}\left[\begin{array}{ll}
-H_{i j} & \left.\left.K_{i j}\right]-\rho\left[\begin{array}{ll}
I & 0 \\
0 & 0
\end{array}\right]\right) \bar{x}
\end{array}\right.\right.
\end{aligned}
$$

The matrices $\Delta Q_{i j}, \Delta L_{i j}$ and $\Delta C_{i j}$ can easily be derived from (1) and the definition of $\operatorname{PWQ}(x)$.

Remark 3. Ideally we would want $\Delta V_{i j}(x) \leq$ $-\rho x^{T} x, \forall x \in \mathcal{P}_{i j}$ and $\Delta V_{i j}(x)$ arbitrary for $x \notin$ $\mathcal{P}_{i j}$. Since this constraint is non-convex, we relax this condition by imposing that $\Delta V_{i j}(x) \leq-\rho x^{T} x$ for all $x$ in a quadratic surface containing $\mathcal{P}_{i j}$ in (8b). This constraint can be made convex by applying the $S$-procedure (Boyd et al., 1994). Since the only constraint on $N_{i j}$ in (8b) is the non-negativity of its elements, the shape of this quadratic surface can be (almost) arbitrarily chosen.

It is now possible to pose the semi-definite program to calculate a PWQ Lyapunov function:

$$
\begin{aligned}
& \text { find } \mathrm{PWQ}_{r}, N_{r}, N_{i j}, \rho, \epsilon, \\
& \text { s.t. } \forall r \in \mathcal{R}, \forall i, j \in \mathcal{T}, \\
& {\left[\begin{array}{rr}
-\Delta Q_{i j}-\rho I-\Delta L_{i j} \\
-\Delta L_{i j}^{T} & -\Delta C_{i j}
\end{array}\right] \succeq\left[\begin{array}{c}
-H_{i j}^{T} \\
K_{i j}^{T}
\end{array}\right] N_{i j}\left[\begin{array}{ll}
-H_{i j} & K_{i j}
\end{array}\right]} \\
& {\left[\begin{array}{cc}
Q_{r}-\epsilon I & \frac{1}{2} L_{r} \\
\frac{1}{2} L_{r}^{T} & C_{r}
\end{array}\right] \succeq\left[\begin{array}{c}
-H_{r}^{T} \\
K_{r}^{T}
\end{array}\right] N_{r}\left[\begin{array}{ll}
-H_{r} & K_{r}
\end{array}\right], \quad(9 \mathrm{~b})} \\
& N_{i j} \geq 0, \quad N_{r} \geq 0, \quad \rho>0, \quad \epsilon>0, \\
& N_{r}=N_{r}^{T}, N_{r} \in \mathbb{R}^{d_{r} \times d_{r}}, N_{i j}=N_{i j}^{T}, N_{i j} \in \mathbb{R}^{d_{i j} \times d_{i j}} \\
& C_{q}=0, \quad L_{q}=0 \in \mathbb{R}^{n}, \\
& \quad \forall q \in \mathcal{R}_{0}, \quad \mathcal{R}_{0} \triangleq\left\{r \in \mathcal{R} \mid 0 \in \mathcal{P}_{r}\right\} . \quad(9 \mathrm{~d})
\end{aligned}
$$

It follows from (8) that (9a) induces $\Delta V_{i j}(x) \leq$ $-\rho x^{T} x$. Inequality $(9 \mathrm{~b})$ ascertains that the PWQ Lyapunov function is bounded from below by a quadratic function and (9c) ensures that all elements of $N_{r}$ and $N_{i j}$ are nonnegative where $d_{r}$ and $d_{i j}$ denote the number of rows of $H_{r}$ and $H_{i j}$, which are defined by $\mathcal{P}_{r}=\left\{x \in \mathbb{R}^{n} \mid H_{r} x \leq\right.$ $\left.K_{r}\right\}$ and (3). Just like for PWA functions (see Remark 2), the quadratic upper bound on the PWQ function does not need to be enforced here, since the Lyapunov function is quadratic around 
the origin (see $(9 d))$ and PWQ on the rest of the state space.

Since equation (9a) is sufficient (not necessary) for $\Delta V_{i j}(x) \leq-\rho x^{T} x$, the SDP formulation is conservative and may not yield a solution even if one exists. The scalar parameters $\epsilon$ and $\rho$ are arbitrarily small but positive in order to enforce a strictly positive PWQ function and exponential stability, respectively.

Theorem 4. (Exponential Stability via SDP). If the SDP (9) associated with an autonomous PWA system of type (1) is feasible, then that system is exponentially stable.

Proof The conditions in (6) are sufficient for exponential stability according to Definition 2, since $S_{\mathrm{PWA}}=\bigcup_{i, j \in \mathcal{T}} \mathcal{P}_{i j}=\bigcup_{i \in \mathcal{R}} \mathcal{P}_{i}$ according to the assumptions in Section 2.1. Therefore we need to show that (9) implies (6). It follows from (8) that (9a) implies (6b). Furthermore (9b) implies that there exists a lower quadratic bound on the PWQ Lyapunov function. A quadratic upper bound exists automatically because of $(9 \mathrm{~d})$. Hence, (9) implies (6).

When computing a common quadratic Lyapunov function $V(x)=x^{T} Q x$ the problem formulation (9) can be drastically simplified. Specifically it is sufficient to impose

$$
\begin{aligned}
& \text { find } Q \succ 0, \rho>0 \text {, } \\
& -\left[\begin{array}{cc}
A_{r}^{T} Q A_{r}-Q+\rho I & A_{r}^{T} Q g_{r} \\
\left(A_{r}^{T} Q g_{r}\right)^{T} & g_{r}^{T} Q g_{r}
\end{array}\right] \\
& \succeq\left[\begin{array}{c}
-H_{r}^{T} \\
K_{r}^{T}
\end{array}\right] N_{r}\left[\begin{array}{ll}
-H_{r} & K_{r}
\end{array}\right], \quad \forall r \in \mathcal{R}, \\
& N_{r} \geq 0, \quad N_{r}=N_{r}^{T}, N_{r} \in \mathbb{R}^{d_{r} \times d_{r}},
\end{aligned}
$$

where $H_{r}$ and $K_{r}$ are defined by (2) and (10c) is used to enforce that each element of the matrix $N_{r}$ is non-negative. In (10), the number of constraints is linear in the number of controller regions $R$ while they are quadratic in (9), when searching for a PWQ Lyapunov function.

\section{PIECEWISE POLYNOMIAL LYAPUNOV FUNCTIONS}

It will be shown in the following how to formulate the search for polynomial or piecewise polynomial Lyapunov function guaranteeing asymptotic stability for autonomous PWA systems by using sumof-squares (SOS) methods (Parrilo, 2003). This issue has been investigated for continuous time PWA systems in (Prajna and Papachristodoulou, 2003).

Before describing the use of SOS for Lyapunov functions, a brief introduction to SOS theory is in order. A multivariate polynomial $p(x)$ is a sum of squares if there exist polynomials $p_{1}(x) \ldots p_{m}(x)$ such that $p(x)=\sum_{i=1}^{m} p_{i}^{2}(x)$. Equivalently,

$$
p(x)=Z(x)^{T} Q Z(x)
$$

where $Z(x)$ is a vector of monomials (e.g. $x \in$ $\mathbb{R}^{2}$ and $Z(x)$ of order $k=2$ implies $Z(x)=$ $\left.\left[\begin{array}{llllll}1 & x_{1} & x_{2} & x_{1} x_{2} & x_{1}^{2} & x_{2}^{2}\end{array}\right]^{T}\right)$ and $Q$ is a positive semidefinite matrix. Being a sum of squares immediately implies non-negativity of $p(x)$, a condition that otherwise is very hard to prove ${ }^{2}$. The computation of a SOS decomposition can be performed using a semidefinite program, which can be solved efficiently. These properties lend themselves very conveniently to the computation of Lyapunov functions. As we move to higher order polynomials, there are more degrees of freedom in choosing the Lyapunov function and this implies that there is a higher probability of finding a Lyapunov function, if it exists. It is also possible to use SOS techniques when applying the $S$-procedure, e.g. it is possible to replace each element in the matrix $N_{r}$ in (9b) with an SOS function. Higher order functions allow for better approximations of the polytopic regions over which the Lyapunov function constraints in (6) are imposed, hopefully leading to further reduction in conservativeness.

Specifically, we aim to find a piecewise polynomial Lyapunov function $\operatorname{PWP}(x)$ of degree $k$, defined by polynomials $\mathrm{PWP}_{r}(x)$ over each polytopic region $\mathcal{P}_{r}$. In the same vein as for the piecewise quadratic case, define $\Delta V_{i j}=\mathrm{PWP}_{j}\left(A_{i} x+g_{i}\right)-$ $\mathrm{PWP}_{i}(x)$. For a stability certificate, we need

$$
\begin{aligned}
& \operatorname{PWP}_{r}(x) \geq \alpha x^{T} x \quad \forall x \in \mathcal{P}_{r}, \forall r \in \mathcal{R}, \\
& \Delta V_{i j}(x) \leq-\rho x^{T} x, \quad \forall x \in \mathcal{P}_{i j}, \forall i, j \in \mathcal{T}
\end{aligned}
$$

In the following, let $G(x)=K-H x$ and $G_{i}(x)$ denote the $i$ th row of $G(x)$. As in the piecewise quadratic case, we can use the $S$-procedure to eliminate the polytopic regions, i.e. add terms of the type $G_{i}(x) N_{i j} G_{j}(x)$ to the constraints. However, nothing prevents us from using higher order multipliers $N_{i j}$, i.e. parameterize the elements $N_{i j}(x)$ as positive polynomials. To allow for even more degrees of freedom, we can also add terms of the form $G_{i}(x) G_{j}(x) G_{k}(x) G_{l}(x)$ and so on. In fact, in can be shown that by using sufficiently many terms and multipliers of sufficiently high order, the higher order $S$-procedure will be both sufficient and necessary (Parrilo, 2003, Th. 5.1).

To calculate a piecewise polynomial Lyapunov function, we apply a higher order $S$-procedure to (12) and replace non-negativity constraints with SOS constraints. The SOS program will be

\footnotetext{
2 Non-negativity does however not imply that the polynomial can be written as a SOS. It is only a sufficient condition for non-negativity
} 


$$
\begin{aligned}
& \operatorname{PWP}_{r}(x)-\alpha x^{T} x=S_{r}(x) \quad \forall r \in \mathcal{R}, \\
& -\rho x^{T} x-\Delta V_{i j}(x)=S_{i j}(x), \quad \forall i, j \in \mathcal{T} .
\end{aligned}
$$

The $S$-procedure terms $S_{q}(x)$ are defined by

$$
\begin{aligned}
& S_{q}(x)=F_{0}^{q}(x)+\sum_{i_{1}=1}^{m} \sum_{i_{2}=1}^{m} F_{i_{1} i_{2}}^{q}(x) G_{i_{1}}(x) G_{i_{2}}(x)+\ldots \\
& +\sum_{i_{1}=1}^{m} \sum_{i_{2}=1}^{m} \ldots \sum_{i_{k}=1}^{m} F_{i_{1} i_{2} \ldots i_{k}}^{q}(x) G_{i_{1}}(x) G_{i_{2}}(x) \ldots G_{i_{k}}(x),
\end{aligned}
$$

where $F_{0}(x)$ is an SOS polynomial of degree $k$, $F_{i_{1} i_{2}}(x)$ of degree $k-2$ and so on and the functions $G_{i_{k}}(x)$ are defined by the sets $\mathcal{P}_{r}$ and $\mathcal{P}_{i j}$ respectively. By constraining all functions $F^{q}(x)$ to be SOS, we can ensure that $S_{q}(x)$ is non-negative if $x \in \mathcal{P}_{q}$. This is a more powerful condition compared to the SDP based $S$-procedure described in the previous section.

The SOS problem for a common polynomial function can be formulated along the same lines as the common quadratic function scheme described in Section 4. We will refrain from a detailed discussion here since the modifications to (10) are straightforward.

Regarding complexity, each SOS condition of degree $k$ involves a vector of monomials $Z(x)$ (see (11)) from degree 1 to $d$, where $d=\frac{k}{2}$. For an $n$ dimensional problem, the total number of monomials is $\left(\begin{array}{c}n+d \\ d\end{array}\right)$. This translates to solving an SDP of size $\left(\begin{array}{c}n+d \\ d\end{array}\right) \times\left(\begin{array}{c}n+d \\ d\end{array}\right)$ (Parrilo, 2003). Every positivity constraint for a region (13a) or decay constraints between 2 regions (13b) is a single such SOS constraint of degree $k$.

Furthermore, each SOS multiplier condition $F^{q}(x)$ (see (14)) of degree $l=0,2 \ldots k$ involves solving an SDP with the size determined by $l$. Consider a constraint of the type (13a) or (13b) over a polytope defined by $m$ half-spaces. There would be $\left(\begin{array}{c}m \\ k-l\end{array}\right)$ SOS multipliers of degree $l$ for this single constraint corresponding to different combinations of $G_{i_{1}}(x) G_{i_{2}}(x) \ldots G_{i_{l}}(x)$, each of which corresponds to an SDP of size $\left(\begin{array}{c}n+l \\ l\end{array}\right) \times\left(\begin{array}{c}n+l \\ l\end{array}\right)$. Note that there is no benefit in choosing the $S$ procedure terms to be of higher order than the Lyapunov function $V(x)$. Ideally, they are of equal order. The rapid growth in problem size places a practical limit on the order of Lyapunov functions (13) and the order of the $S$-procedure terms (14) which can be computed for medium sized PWA systems (i.e. several hundred regions).

\section{TUNING PARAMETERS}

As stated in the previous sections, the complexity of the various Lyapunov function computation schemes can be prohibitive for large partitions. This will also be illustrated by the case study in Section 7. Hence, this section will discuss modifications to the previously introduced problem formulations which make the associated computations more efficient.

Fixed Exterior Ellipsoids: The standard $S$ procedure described in Section 4 achieves the objective of ensuring positivity over a particular region by approximating that region with a quadratic surface (see (8b)). This version of the $S$-procedure is not lossless (Boyd et al., 1994), but the shape of the quadratic surface is a degree of freedom in the solution. As an alternative, it is possible to fix the surface a priori, i.e. by selecting it to be the minimal volume ellipsoid containing the region. The associated $S$-procedure is lossless and the degrees of freedom in the SDP are significantly reduced. The new problem formulation is given by

$$
V(x)-\tau\left[\begin{array}{cc}
-E & E x_{0} \\
x_{0}^{T} E^{T} & 1-\left(x_{0}^{T} E x_{0}\right)
\end{array}\right] \geq 0
$$

where the minimum volume exterior ellipsoid is defined by $\left(x-x_{0}\right)^{T} E\left(x-x_{0}\right) \leq 1$. Here, there is just one free variable $\tau$, compared to the $\frac{m(m-1)}{2}$ free variables in $(8 \mathrm{~b})$, where $m$ is the number of half-spaces defining the polytope.

However, as stated in (Johansson, 2002), using the exterior ellipsoid is always more conservative than using the $S$-procedure in (8b). Therefore, for simple partitions, the ellipsoidal approach may be more of a liability due to the effort required for the calculation of the exterior ellipsoid and the increased conservativeness. The computational advantages are more discernible for partitions with a large number of regions, for which a significant reduction in the number of decision variables is achieved.

Upper Bound Constraints: The upper bound constraints in Theorems 1 and 2 can be omitted by enforcing a certain structure upon the Lyapunov function around the origin. For example, when searching for PWQ functions it is sufficient to enforce that the function has no affine and offset terms $\left(L_{r}=0, C_{r}=0\right)$ for the regions containing the origin $\left(0 \in \mathcal{P}_{r}\right)$ and for PWA functions it is sufficient to enforce that the function has no offset terms $\left(C_{r}=0\right)$ for the regions containing the origin $\left(0 \in \mathcal{P}_{r}\right)$. If this structure is imposed, it follows trivially that an upper bound on $V(x)$ exists, and the associated constraints can be omitted.

Lower Bound Constraints: In many practical cases, it is advisable to completely discard the lower bound constraints for regions that do not contain the origin (Johansson, 2002). For 
asymptotically stable systems, the decay constraint will directly imply that a lower bound exists. Since this is not true for unstable systems, it is necessary to check the existence of a lower bound in the second step. Since the complexity of SDP solvers is polynomial this 'divide-and-conquer' approach will guarantee faster runtime.

Degree of SOS Multipliers: In SOS-schemes, the degree of the polynomial functions is a crucial influence on the likelihood of finding a Lyapunov function. In order for the constraints in (13) not to be too conservative, it is advisable to select the Lyapunov polynomial to be of the same order as the associated $S$-procedure terms. However, as we increase the degree of the SOS functions, the number of variables in the associated SDP problem increases fast, placing a practical limit on tractable problem sizes. Hence, it may sometimes be advisable to select the $S$-procedure multipliers of a lower order than the associated Lyapunov function, in order to keep the degrees of freedom limited.

\section{CASE STUDY}

\subsection{Problem Setup}

The systems considered in our case study are constrained LTI and PWA systems subject to optimal PWA control. Our case studies were performed on a large number of random open-loop stable and unstable systems of order 2 and 3 with one input. The PWA systems were created by assuming random dynamic matrices defined over four random non-overlapping polytopes, whose union covers the feasible state space.

The elements of the dynamic system matrices were assigned random values between -2 and +2 and the system inputs and states were constrained

$$
\|u(k)\|_{\infty} \leq 1 \text { and }\|x(k)\|_{\infty} \leq 10, \forall k \geq 0 .
$$

The control objective was defined by a finite horizon cost,

$$
J^{*}(x(k))=\sum_{k=0}^{N-1} \min _{u}\left\|Q_{x} x(k+1)\right\|_{p}+\left\|Q_{u} u(k)\right\|_{p}
$$

using both the standard squared Euclidean norm $(p=2)$ and linear norms $(p=1$ and $p=\infty)$. The weights were always $Q_{u}=10 I$ and $Q_{x}=$ I. Calculation of an explicit solution to these optimal control problems all result in a PWA control law, formalized in the following theorem (Borrelli, 2003; Bemporad et al., 2002).

Theorem 1. Consider an optimal control problem (16) for an LTI system subject to constraints
(15). For the set of feasible parameters $\mathcal{X}_{N}$, the optimizer $U_{N}^{*}: \mathcal{X}_{N} \rightarrow \mathbb{R}^{N}$ is piecewise affine (PWA), i.e.

$$
U_{N}^{*}(x)=F_{r} x+G_{r}, \quad \text { if } \quad x \in \mathcal{P}_{r}
$$

where $\mathcal{P}_{r}=\left\{x \in \mathbb{R}^{n} \mid H_{r} x \leq K_{r}\right\}, r=1, \ldots, R$.

As a consequence of this theorem, the closed loop systems we are analyzing in this case study are all constrained autonomous PWA systems.

Specifically, we applied the control scheme in (Grieder and Morari, 2003; Grieder et al., 2004) such that invariant PWA partitions were obtained. This is achieved by posing a receding horizon control problem with an invariance constraint on the first state, i.e. the state at time $k+1$ is restricted to be contained inside the maximal control invariant set. Although the obtained PWA partitions are guaranteed to be invariant, there is no guarantee of asymptotic stability. Therefore, the schemes in (Grieder and Morari, 2003; Grieder et al., 2004) rely heavily on the stability analysis of PWA systems investigated here.

The PWA partitions considered here were obtained for prediction horizons $N=1,3,5$. The partitions consisted of 9 to 201 regions with 9 to 515 associated transitions. We chose relatively small systems since this allowed us to perform the case study on a large number of systems within a reasonable amount of time. The large runtime for the SOS based methods did not allow us to consider partitions with a larger number of regions, as will be illustrated in Section 7.3.

We will now briefly motivate our selection of systems which we analyzed. The choice of systems was mainly driven by two objectives: the stability analysis must have practical relevance and the PWA partition must be invariant, as discussed in Section 2.1. Both of these objectives are naturally met by the controller partitions considered here. In addition, the control objective (16) was selected such that the expensive control action $\left(Q_{u}>Q_{x}\right)$ may easily lead to unstable closedloop behavior. Finally, the scheme in (Grieder and Morari, 2003; Grieder et al., 2004) yields PWA systems of relatively low complexity. Therefore, the systems used in this case study are a good choice for the above stated reasons of invariance, practical relevance and low complexity. Note that it is not possible to consider random bounded PWA partitions directly since these will not be invariant, in general.

All computations were carried in MATLAB, using the Multi-Parametric Toolbox (Kvasnica et al., 2003) and YALMIP (Löfberg, 2004). 


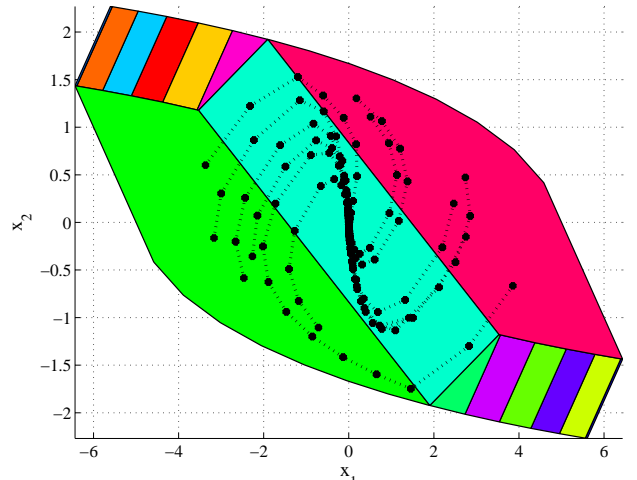

(a) $N=1$, Initial state is close to origin: Convergent Trajectories.

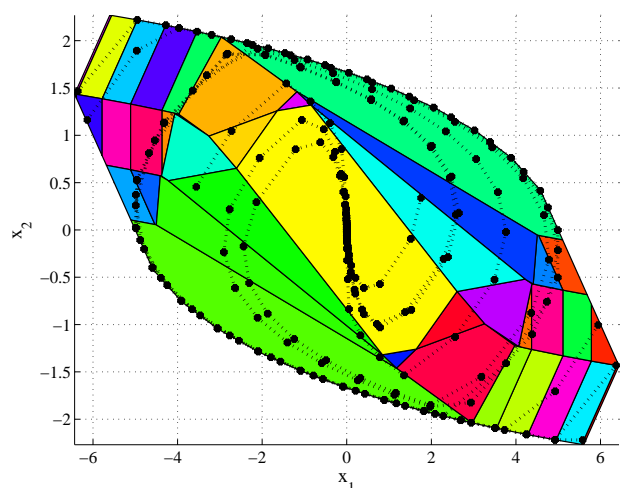

(c) $N=3$

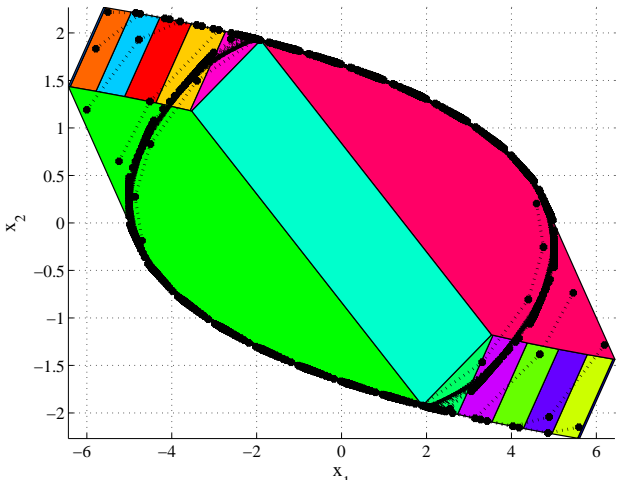

(b) $N=1$, Initial state is far from origin: Limit Cycle Trajectories.

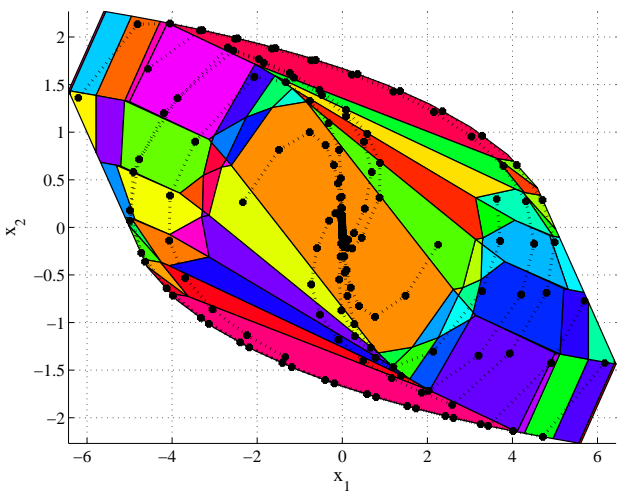

(d) $N=5$

Fig. 1. Closed-loop trajectories for Example 1 for various prediction horizons $N$.

\subsection{Numerical Results - Specific Systems}

Before presenting the results of the large scale case study in Section 7.3, we will focus on specific systems which exhibit certain properties that we wish to highlight.

Example 1. Consider the unstable $2^{\text {nd }}$ order system with one input defined by

$$
x(k+1)=\left[\begin{array}{cc}
1.2 & 1.2 \\
0 & 1.2
\end{array}\right] x(k)+\left[\begin{array}{c}
1.0 \\
0.5
\end{array}\right] u(k)
$$

The system is subject to the constraints $\|x(k)\|_{\infty} \leq$ 5 and $\|u(k)\|_{\infty} \leq 1, \forall k \geq 1$. The control objective in (16) is defined by the 2-norm and the weights $Q_{x}=I$ and $Q_{u}=1$.

If we apply an optimal controller as in (Grieder and Morari, 2003) with prediction horizon $N=1$ to Example 1, all stability analysis schemes considered here (PWA, PWQ, piecewise SOS up to fourth order) fail. When simulating closed loop trajectories, one can observe that the system converges to the origin if the initial state is close to the origin, see Figure 1(a). However, if the initial state is further away, the system reaches a limit cycle, as is depicted in Figure 1(b). Hence, the system is indeed not asymptotically stable.
If we increase the prediction horizon to $N=3$, the convergent closed-loop trajectories in Figure 1(c) are obtained. However, none of the techniques considered here succeeds in finding a Lyapunov function. If the prediction horizon is increased to $N=5$, the system is stable as can be seen from the trajectories in Figure 1(d). For the resulting partition, it is not possible to find common quadratic or common quartic Lyapunov functions, while piecewise quadratic and piecewise quartic functions can be computed.

This simple example clearly illustrates the conservativeness of certain types of Lyapunov functions as well as the impact of the controller prediction horizon $N$ on stability of the closed-loop system.

Figure 2 shows the plots of different Lyapunov functions for the PWA partition which is obtained when applying the control scheme in (Grieder and Morari, 2003) with prediction horizon $N=1$ to the following stable LTI system:

$$
x(k+1)=\left[\begin{array}{cc}
0.4734 & 0.6756 \\
0.7353 & -0.1321
\end{array}\right] x(k)+\left[\begin{array}{c}
0.4776 \\
0.4459
\end{array}\right] u(k)
$$




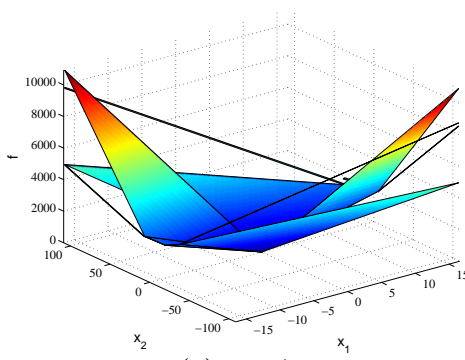

(a) PWA

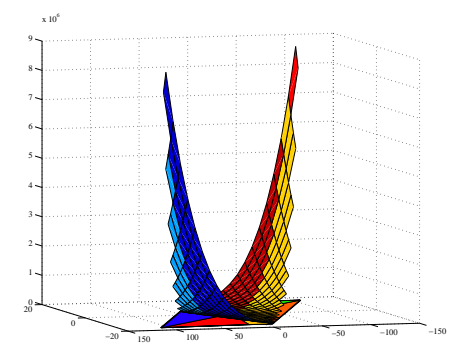

(d) Common SOS order 4

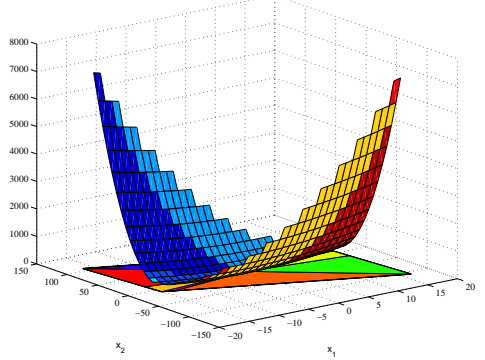

(b) Quadratic

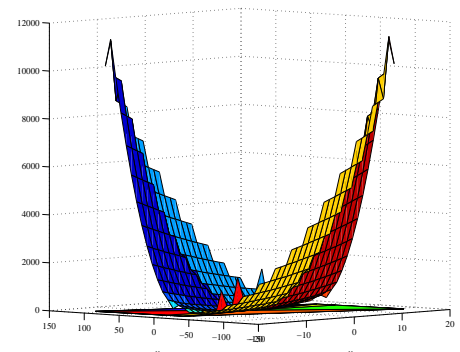

(e) Piecewise SOS order 2

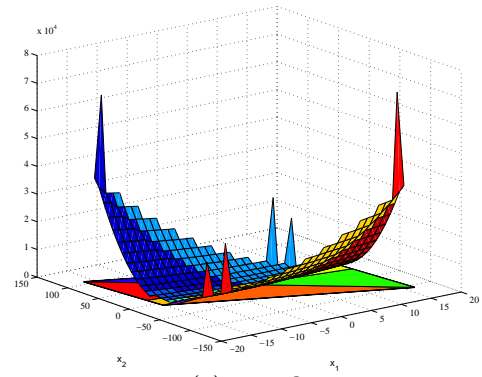

(c) PWQ

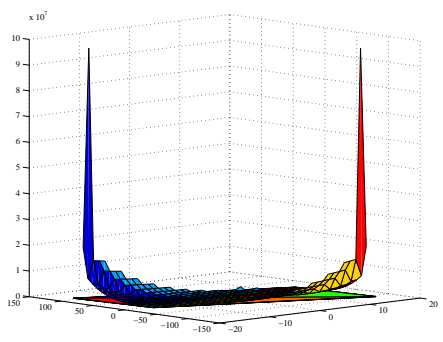

(f) Piecewise SOS order 4

Fig. 2. Different Lyapunov functions for same controller

\subsection{Numerical Results - Random Systems}

The results in this section were obtained by considering 100 random PWA systems and 200 random LTI systems subject to the control scheme in (Grieder and Morari, 2003; Grieder et al., 2004) for prediction horizons $N=1,3$ and 5. Specifically, the likelihood of successfully computing a Lyapunov function as well as the associated computation time are given in Tables 1-3.

The solution time corresponds to the time spent in computing the solution to the problems as described in the previous sections. The setup time refers to the time required to do the pre-processing (e.g. reachability analysis, vertex enumeration) and actual setup of the constraints. All the SOS data provided in the following tables were obtained with YALMIP (Löfberg, 2004). See the discussion in Section 7.4 for details on the choice of solvers. Note that we have also used the SOS based code to compute both common quadratic and PWQ Lyapunov functions, for verification reasons. The computation times and success rates were consistent with the results obtained with the methods in Section 4 and are therefore not restated here.

If no Lyapunov function could be found with any method, we analyzed the corresponding partition through exhaustive simulation. Amongst all the analyzed partitions for the LTI systems, for less than $1 \%$ of the systems that exhibited convergent trajectories, the Lyapunov analysis failed. For the PWA systems however, we were unable to find a
Lyapunov function for nearly $10 \%$ of the systems that exhibited convergent trajectories.

\subsection{Discussion of Results}

\subsubsection{Likelihood of Success}

- If the control scheme in (Grieder and Morari, 2003) is applied to stable LTI systems, the stability analysis of the resulting PWA systems is very likely to be successful. There are cases where quadratic and PWA approaches fail but the PWQ approach succeeds. On the other hand, we have also observed cases where the PWA approach succeeds while the quadratic and PWQ approach fails.

- The stability analysis of the PWA systems generated by using the control scheme in (Grieder et al., 2004) for PWA systems has a higher possibility of failure. The failure rate of common quadratic and higher order polynomial approaches is the highest. It is much easier to find a Lyapunov function using piecewise techniques. This behavior coincides with our expectations, since the likelihood of finding a common Lyapunov function over completely different dynamics is relatively low.

- It is interesting to observe that the number of convergent closed-loop systems resulting from unstable LTI systems is much lower for linear performance objectives, even though the associated prediction horizons are much larger than for the quadratic objectives 


\begin{tabular}{|c|c|c|c|c|c|c|}
\hline \multicolumn{7}{|c|}{ Partitions obtained for $2^{\text {nd }}$ order PWA systems, $N=1$} \\
\hline & \multicolumn{2}{|c|}{50 Systems, $\infty$ norm objective } & \multicolumn{3}{c|}{50 Systems, 1 norm objective } \\
\hline Method & Success & Solution Time & Setup Time & Success & Solution Time & Setup Time \\
\hline Quadratic & $14 / 45$ & $1.3 \mathrm{sec}$. & $1.7 \mathrm{sec}$. & $6 / 46$ & 0.9 sec. & $1.4 \mathrm{sec}$. \\
Piecewise Affine & $42 / 45$ & $0.9 \mathrm{sec}$. & $7.7 \mathrm{sec}$. & $40 / 46$ & 1.9 sec. & 13.1 sec. \\
Piecewise Quadratic & $43 / 45$ & $6.4 \mathrm{sec}$. & $9.9 \mathrm{sec}$. & $37 / 46$ & 7.0 sec. & 10.8 sec. \\
Common SOS order 4 & $19 / 45$ & $0.9 \mathrm{sec}$. & $0.4 \mathrm{sec}$. & $9 / 46$ & 0.7 sec. & 0.3 sec. \\
Piecewise SOS order 4 & $36 / 45$ & $54.0 \mathrm{sec}$. & $21.3 \mathrm{sec}$. & $22 / 46$ & $55.2 \mathrm{sec}$. & $23.3 \mathrm{sec}$. \\
\hline
\end{tabular}

Table 1. The number of regions were between 29 and 201 with 63-515 transitions.

'Success' denotes the number of Lyapunov functions found out of the total number of systems with convergent trajectories, the 'Solution Time' is the cpu-time required to solve the associated optimization problem and 'Setup Time' is the time needed to pre-process the problem.

\begin{tabular}{|c|c|c|c|c|c|c|}
\hline \multicolumn{6}{|c|}{ Partitions obtained for $3^{\text {rd }}$ order LTI systems, 2 norm objective } \\
\hline & \multicolumn{3}{|c|}{50 Stable Systems, $N=1$} & \multicolumn{3}{|c|}{50 Unstable Systems, $N=1$} \\
\hline Method & Success & Solution Time & Setup Time & Success & Solution Time & Setup Time \\
\hline Quadratic & $45 / 50$ & 0.7 sec. & 0.4 sec. & $43 / 50$ & 1.1 sec. & 0.5 sec. \\
Piecewise Quadratic & $50 / 50$ & 1.9 sec. & 1.0 sec. & $50 / 50$ & 2.9 sec. & 1.5 sec. \\
Common SOS order 4 & $50 / 50$ & 2.2 sec. & 0.7 sec. & $50 / 50$ & 3.4 sec. & 1.7 sec. \\
Piecewise SOS order 4 & $40 / 50$ & 8.2 sec. & 4.3 sec. & $13 / 50$ & 21.5 sec. & 9.3 sec. \\
\hline
\end{tabular}

Table 2. The number of regions were between 9 and 15 with 9-47 transitions.

'Success' denotes the number of Lyapunov functions found out of the total number of systems with convergent trajectories, the 'Solution Time' is the cpu-time required to solve the associated optimization problem and 'Setup Time' is the time needed to pre-process the problem.

\begin{tabular}{|c|c|c|c|c|c|c|}
\hline \multicolumn{4}{|c|}{ Partitions obtained for unstable $2^{\text {nd }}$ order LTI systems, $\infty$ norm objective } \\
\hline & \multicolumn{3}{|c|}{50 Systems, $N=3$} & \multicolumn{3}{c|}{50 Systems, $N=5$} \\
\hline Method & Success & Solution Time & Setup Time & Success & Solution Time & Setup Time \\
\hline Quadratic & $20 / 28$ & 1.6 sec. & 1.9 sec. & $17 / 17$ & 2.8 sec. & 3.9 sec. \\
Piecewise Affine & $20 / 28$ & 4.8 sec. & 22.6 sec. & $17 / 17$ & 10.3 sec. & 45.5 sec. \\
Piecewise Quadratic & $26 / 28$ & 6.7 sec. & 12.7 sec. & $17 / 17$ & 11.1 sec. & 24.2 sec. \\
Common SOS order 4 & $28 / 28$ & 1.7 sec. & 1.1 sec. & $17 / 17$ & 1.8 sec. & 1.1 sec. \\
Piecewise SOS order 4 & $23 / 28$ & 43.5 sec. & 24.0 sec. & $16 / 17$ & 97.7 sec. & 52.2 sec. \\
\hline
\end{tabular}

Table 3. For $N=3$ the number of regions was between 40 and 72 with $56-154$ transitions. For $N=5$ the number of regions was between 70 and 184 with 100-363 transitions. 'Success' denotes the number of Lyapunov functions found out of the total number of systems with convergent trajectories, the 'Solution Time' is the cpu-time required to solve the associated optimization problem and 'Setup Time' is the time needed to pre-process the problem.

(see Tables 2 and 3). The cause of this is not apparent, but part of ongoing research. The large number of convergent closed-loop systems which were obtained by controlling PWA systems (see Table 1) is attributable to the fact that not all of the random PWA systems were unstable. Note that it is impossible to generate random unstable PWA systems.

- Theoretically, a higher order $S$-Procedure as illustrated in (14) should have a positive influence on the likelihood of successful analysis, since it allows for higher order approximations of the polytopic regions. In practice, however, higher order multipliers result in more frequent numerical problems and the likelihood of successful analysis is decreased. In hundreds of simulations we have not been able to find a PWA partition where the SOS approaches outperform the lower order SDP schemes.

- It was observed that the numerical problems associated to the SOS approaches occur regardless of SOS (Löfberg, 2004; Prajna et al., 2004) and SDP (Sturm, 1999; Toh et al., 1999) solver. Problem specific solvers or novel problem formulations may be able to alleviate the numerical issues. We have not been able to satisfactorily deal with the numerical issues resulting from the SOS problems. As a consequence, the success rates of 
the SOS approaches remained disappointing.

- Recovering from numerical problems in the optimization problems and dealing with the associated low quality solutions, is much more straightforward for linear and quadratic Lyapunov functions, compared to SOS based. The reason is that validity of a quadratic Lyapunov function easily can be checked a-posteriori by, e.g., checking eigenvalues. Checking the validity of slightly perturbed SOS decompositions is however much more intricate. This problem is part of on-going research and has already motivated the introduction of a post-processing algorithm in the SOS module in YALMIP (Löfberg, 2004).

- Using the minimum volume exterior ellipsoids to reduce the number of variables has a surprisingly strong impact on the likelihood of successful analysis, i.e. it is much harder to find Lyapunov functions.

- Overall, it was observed that the simpler functions like common quadratic, PWA, PWQ were most effective in obtaining a certificate of stability for the type of problems considered here. Overall, the percentage of systems exhibiting convergent behavior for which no Lyapunov function was found is below $5 \%$.

\subsubsection{Computation Time}

- The overall computation time correlates directly to the number of regions and, more importantly, to the number of transitions which occur between regions. In general, unstable LTI systems result in more complex partitions such that the associated stability analysis is more time consuming.

- Since the PWA Lyapunov function computation requires vertex enumeration of the polytopes defining regions and transitions, the problem setup time is larger than for the quadratic and PWQ case.

- In general, the linear cost objectives generate partitions consisting of more regions than those obtained for quadratic cost objectives. Hence, the associated analysis is more time consuming.

- The piecewise SOS scheme, although being theoretically more powerful than the other techniques has practical limitations. As is clear from the tables, much of the computation time is spent in setting up the problem. The reason is the large amount of symbolic manipulation of higher order polynomi- als needed for the SOS formulations.

- Neglecting the lower bound constraints in the problem formulation as described in Section 6 leads to large speedups, especially in the SOS based cases.

- Exterior Ellipsoids: For the partition sizes considered here, no runtime benefit was obtained by computing the exterior ellipsoids a priori. A benefit may result for larger partitions.

\section{CONCLUSION}

An extensive survey of various methods of computing Lyapunov functions for discrete-time PWA systems was presented in this paper. First, the basic building blocks (e.g. reachability analysis) and assumptions (e.g. set invariance) were established. Second, it was shown how to compute PWA, PWQ and higher order piecewise polynomial Lyapunov functions for discrete-time systems. The computation schemes for PWA and higher order piecewise polynomial functions were not previously published for discrete-time PWA systems.

Finally, the results of an extensive case study are given. The PWA systems considered here are the result of applying the control scheme in (Grieder and Morari, 2003; Grieder et al., 2004) to random LTI and PWA systems. We consider the resulting autonomous PWA systems to be a good choice for our case study because of invariance of the controlled set, practical relevance and low complexity of the partition. Note that it is not possible to consider random bounded PWA partitions directly since these will not be invariant, in general. Unbounded PWA systems were not considered due to the limited practical relevance. The case study illustrates that simple Lyapunov functions (i.e. quadratic, PWA or PWQ) are generally sufficient for analyzing discrete-time PWA systems of the type considered here.

All tools as well as the random systems be downloaded from (Kvasnica et al., 2003).

\section{REFERENCES}

Bemporad, A. (2004). Efficient conversion of mixed logical dynamical systems into an equivalent piecewise affine form. IEEE Trans. Automatic Control 49(5), 832-838.

Bemporad, A. and M. Morari (1999). Control of systems integrating logic, dynamics, and constraints. Automatica 35(3), 407-427.

Bemporad, A., G. Ferrari-Trecate and M. Morari (2000). Observability and controllability of piecewise affine and hybrid systems. IEEE Trans. Automatic Control 45(10), 1864-1876.

Bemporad, A., M. Morari, V. Dua and E.N. Pistikopoulos (2002). The explicit linear quadratic regulator for constrained systems. Automatica 38(1), 3-20. 
Borrelli, F. (2003). Constrained Optimal Control Of Linear And Hybrid Systems. Vol. 290 of Lecture Notes in Control and Information Sciences. Springer.

Boyd, S., L. El Ghaoui, E. Feron and V. Balakrishnan (1994). Linear Matrix Inequalities in System and Control Theory. Studies in Applied Mathematics. SIAM.

Feng, G. (2002). Stability analysis of piecewise discretetime linear systems. IEEE Trans. on Automatic Control 47(7), 1108-1112.

Ferrari-Trecate, G., F. A. Cuzzola, D. Mignone and M. Morari (2002). Analysis of discrete-time piecewise affine and hybrid systems. Automatica 38(12), 21392146.

Grieder, P. and M. Morari (2003). Complexity reduction of receding horizon control. In: Proc. 42th IEEE Conf. on Decision and Control. Maui, Hawaii, USA.

Grieder, P., M. Kvasnica, M. Baotić and M. Morari (2004). Low complexity control of piecewise affine systems with stability guarantee. In: Proc. of the American Control Conference. Boston, USA.

Grieder, P., M. Lüthi, P. Parillo and M. Morari (2003a). Stability \& feasibility of receding horizon control. In: European Control Conference. Cambridge, UK.

Grieder, P., P. Parillo and M. Morari (2003b). Robust receding horizon control - analysis \& synthesis. In: Proc. 42th IEEE Conf. on Decision and Control. Maui, Hawaii, USA.

Heemels, W.P.M.H., B. De Schutter and A. Bemporad (2001). Equivalence of hybrid dynamical models. $A u$ tomatica 37(7), 1085-1091.

Johansson, M. (2002). Piecewise Linear Control Systems A Computational Approach. Vol. 284 of Lecture Notes in Control and Information Sciences. Springer.

Johansson, M. and A. Rantzer (1998). Computation of piece-wise quadratic Lyapunov functions for hybrid systems. IEEE Trans. Automatic Control 43(4), 555559.

Kvasnica, M., P. Grieder, M. Baotić and M. Morari (2003). Multi Parametric Toolbox (MPT). In: Hybrid Systems: Computation and Control. Lecture Notes in Computer Science, Volume 2993. Springer Verlag. Pennsylvania, Philadelphia, USA. pp. 448-462. http: //control.ee.ethz.ch/ mpt.

Löfberg, J. (2004). YALMIP : A toolbox for modeling and optimization in MATLAB. In: Proceedings of the CACSD Conference. Taipei, Taiwan. Available from http://control.ee.ethz.ch/ joloef/yalmip.php.

Parrilo, P.A. (2003). Semidefinite programming relaxations for semialgebraic problems. Mathematical Programming Ser. B 96(2), 293-320.

Prajna, S., A. Papachristodoulou, P. Seiler and P. A. Parrilo (2004). SOSTOOLS: Sum of squares optimization toolbox for MATLAB.

Prajna, S. and A. Papachristodoulou (2003). Analysis of switched and hybrid systems - beyond piecewise quadratic methods. In: American Control Conference. Denver, Colorado, USA.

Sontag, E.D. (1981). Nonlinear regulation: The piecewise linear approach. IEEE Trans. Automatic Control 26(2), 346-358.

Sontag, E.D. (1996). Interconnected automata and linear systems: A theoretical framework in discrete-time. In: Hybrid Systems III - Verification and Control (R. Alur, T.A. Henzinger and E.D. Sontag, Eds.). pp. 436-448. Number 1066 In: Lecture Notes in Computer Science. Springer-Verlag.

Sturm, J.F. (1999). Using SeDuMi 1.02, a MATLAB toolbox for optimization over symmetric cones. Optimization Methods and Software 11-12(1-4), 625-653.

Toh, K. C., M. J. Todd and R. H. Tütüncü (1999). SDPT3 - a Matlab software package for semidefinite programming, version 2.1. Optimization Methods and Software 11-12(1-4), 545-581.

Torrisi, F.D. and A. Bemporad (2004). HYSDEL - a tool for generating computational hybrid models. IEEE Trans. Contr. Sys. Techol. 12, 235-249. http:// control.ee.ethz.ch/ hybrid/hysdel/hysdel.msql.

Vidyasagar, M. (1993). Nonlinear Systems Analysis. 2nd ed.. Prentice Hall. 